\title{
Interoperability Challenges of ERP Implementation in a Collaborative Manufacturing Environment
}

\author{
Yongsheng $\mathrm{Ma}^{1, \text { a }}$ \\ ${ }^{1}$ Department of Mechanical Engineering, University of Alberta, Edmonton, AB T6G 2G8, Canada \\ ayongsheng.ma@ualberta.ca
}

Keywords: ERP; Engineering informatics; Unified feature modeling; Low volume and high mix production; Information system interoperability

\begin{abstract}
The paper tries to address the common challenges that many small and medium enterprises (SMEs) are facing: the manufacturing data integration with a collaborative manufacturing environment with low volume and high mix production orders. The research focus is on the interoperability between the existing and a new ERP system, as well as the collaboration scheme among different suppliers and partners in a supply chain model.
\end{abstract}

\section{Introduction}

The global competition trend has forced industries to implement advanced engineering management information technology like using Enterprise Resources Planning (ERP) software tools for manufacturing operation management. Corporations or organizations must be able to react rapidly and effectively against the changes of market need. Using an ERP software system will help them reducing development cycle time, which has an important role in the competitive market. An ERP system is intended to automate parts or all of business activities, from order generation, production planning, process planning, work shop scheduling, supply chain management, inventory control, order delivery, and in field services with an integrated software platform. An ERP system usually has several modules such as Order Management, Financial management, Capacity Management, Scheduling and Planning, Process Planning, Knowledge Management, Customer Relation or Project Management, Product Management, and Service Management, etc. It should be pointed out that implementation of ERP involves high failure rate because of the mismatch of the technology selected and the actual production processes, inadequate re-engineering strategies and the lack of research and development technical capability [1]. Traditionally, in ERP implementation, standard processes are adopted in order to reduce the business risks [2, 3] which companies are most concerned with.

Currently, many small and medium enterprises (SMEs) are moving towards reconfigurable manufacturing systems $[4,5]$. This is due to the fact that there are excessive production capability in the market and massive economic globalization. In the global market, supply chain oriented systems are more and more implemented recently as a new manufacturing paradigm aiming at short lead time, more product variants, low and fluctuating volumes and low prices. In essence, such a system is a virtual manufacturing system with more complex production planning and control that is developed with advanced informatics software engineering effort [5].

This business transition imposes even ERP research and development challenges to companies. In collaborative manufacturing environment, each company has lots of internal and external information that needs to managed and then used efficiently. With this new business requirement, manufacturing procedures need to be modeled in a generic manner so that the data structures generated by, or developed in, individual ERP tools can be seamlessly integrated with, or migrated into a common data structure which is in turn to be interfaced with a collaborative ERP tool. So far, the research on system interoperability between different ERP systems in the transition from one 
business model to another is a highly challenging research focus. There is no existing and systematic research work has been reported. The required information system supporting new collaborative manufacturing should be able to integrate different companies' manufacturing planning and control systems as an integrated supply chain network.

This paper is introduces the scalable interoperability technology for upgrading Enterprise Resources Planning (ERP) applications in the critical business transition, i.e. from self-dependent and in-house manufacturing to an open and global supply chain based production. The effort reported here is continued research collaboration between University of Alberta and a Canadian medium size oil drilling equipment manufacturer.

\section{Research Challenges Identified}

Four new research challenges are identified by the author: (1) To deal with the interoperability issues among other CAx systems (among engineering design tools, modules of old and new ERP systems); (2) Manufacturing planning and control transition from in-house manufacturing to supply chain oriented production, (3) A neutral data model for ERP upgrading (between two different packages), and (4) cost engineering.

For the first point, it can be appropriated that customer-specific product orders are associated to complex product assembly structures and configurations. To address the diversity of product orders, dynamic product driven manufacturing processes have to be modeled in a generic manner. The research focus is on the interoperability between the existing product engineering models and a federation of ERP systems, as well as the collaboration scheme among different suppliers and partners in a supply chain model. Dynamic capacity modeling method has to be developed, then followed by a working computer program that is designed to generate and modify coordinated master production schedule with the consideration of available capabilities and resources. The research solution framework should be aimed to investigate a systematic data interoperability mapping method that enables the system to not only control production for a specific product order configuration, but also simulate "what-if" scenarios before implementing a solution. The dynamic "what-if" scenario search function would provide a "simulation" capability for the company to go through a "fine-grain" virtual trial of production so that the customer order quote and delivery time could be given timely and confidently. To my knowledge the existing ERP systems could not achieve such "accurate" details.

Secondly, research effort is required in generic manufacturing process modeling and redevelopment of ERP interfaces for the new scenarios with the distributed manufacturing management requirement; generic computational solution has to be available for the plug-and-pay resources planning and job scheduling in a dynamic supply chain system. Currently, many companies have a long "frozen time period" that hurdles responsiveness of a manufacturing company. Generic planning and scheduling model must considers the real complexity of collaboration involved in making a master plan when each resource capacity is limited and when products have flexibility of being produced at different settings. In most existing ERP solutions, the mater planning and scheduling module (MPS) produces production schedules in a finite planning horizon, assuming infinite capacity, fixed processing times, and deterministic demand; the disadvantages are well studied by researchers in the field. To support such collaboration, some imperative research deliverables are the generic manufacturing outsourcing and in-house processing models with individual partners' fine-grain resources capacity definitions. 
Thirdly, to achieve the ERP interoperability, a neutral ERP process data model has to be developed which include two parts: (1) neutral data schema definition for the automated ERP data migration or integration; (2) procedures in the form of standardized operation forms and computerized management interfaces that can be integrated with the different ERP systems. Currently, an ERP package is a software giant covering only the process domain of the implemented company. However, ERP software has very poor interoperability with other ERP tools because there is no generically designed "neutral" process model available in the world, unlike the case that there is a STEP standard (ISO) in the product domain. Any new development in this filed will be a great advancement in engineering informatics interoperability [6]. This is an ambitious scientific research direction in engineering informatics field. This research direction will generate a new momentum in the "standardization" of process models with different manufacturing environments.

Fourthly, a cost engineering method under the new framework of ERP implementation has to be developed.

\section{Proposed Solution Framework}

The proposed solution framework consists of four essential aspects. First, to enable the required flexibility in ERP implementation that can be catered for small batch and high diversity production, advanced semantic modeling and data integration between the product and process domains must be carried out. Customers need an assurance on the feasibility of their product configurations and the accurate delivery time for their confirmed orders. Before really gaining experience by testing all the "real-world" processes, the best bet by the company is to evaluate the product configuration automatically by checking the design and manufacturing constraints and to forecast the cost and delivery time virtually with some algorithms. One of the enabling blocks of knowledge in such a potential forecasting technology is the associative connection between customer product configuration feature model, which is embedded in the form of customer features organized via customers, and precise and accurate manufacturing cost and capacity models; and the author believes unified feature modeling is the key to solve this problem [7, 8]. For example, as to manufacturing capacity modeling, the corresponding capacity features can be defined according to the required product features for all manufacturing resources, such as machines.

Two new feature-based domains were proposed that are to be incorporated into new ERP process modeling: Customer domain and Capacity domain [7]. Both of them are associative extensions of the integrated product and process domains. The customer domain relates customer requirements, quotes, and orders with customer-specific product configuration features, and further translates those product configurations into specific production process features. Such feature-based association method is new. This new concept will support the "dynamic supply chain system" as we have described in the proposal. To the author's best knowledge, the existing ERP systems could not achieve such "accurate" details. A simple example could highlight the importance of fine grain feature level analysis for the resources planning. For example, a company has a CNC lathe, but its machine cutters do no cover all the operations required for a part, which demands all the operations are done under one setup to assure the GDT tolerances specified; or the lathe is not stiff enough to stand the cutting forces expected; or the tooling of locating and clamping does not fit. Without detailed capacity feature checking, that part could be assigned to the CNC lathe but eventually could not be machined due to the lack of machining capacity.

Second, this paper suggests investigating a systematic data interoperability mapping method that enables the system to not only control production, but also simulate "what-if" scenarios before implementing a solution." We would refer to our recent IEEE paper to explain more details [7]. The dynamic "what-if" scenario search function would provide a "simulation" capability for the 
company to go through a "fine-grain" virtual trial of production so that the customer order quote and delivery time could be given timely and confidently. We propose a new type of features - capacity features $[7,8]$ for all the resources. Note that the matching of the capacity of a resources to a product configuration feature is necessary due to the specific interpretation of "capacity" subject to the targeting feature set during the run time. This new concept will support the "dynamic supply chain system" as we have described in the proposal.

In the current ERP application, it has been frequently encountered that, imprecise machine capacity description causes the ambiguous job assignment and creates uncertainty. It is pretty common that the machine intended to do the job is not able to fulfill the technical requirement such as geometrical and dimensional tolerances, stiffness required, or simply the machining envelope. Capacity feature $[7,8]$ is assumed to be dynamically generated and evaluated according to the required operation and the specific work piece condition. Such dynamic matching mechanism is a new concept that has been developed by the $U$ of $A$ research team and the modeling paradigm is going to be further investigated in the near future.

Third, a neutral process informatics model is proposed which can be the cornerstone for semantic modeling towards generic manufacturing process data interoperability. Here, the "neutral process data model" means that the process data structures and constraints that are generically and comprehensively modeled in such a way that different ERP packages can communicate with the potential process interaction module without data recognition problem. Such exchangeability in a less integrated environment, or the "interoperability" in more advanced sense, is necessary for managing an active manufacturing collaboration network. Based on our findings from previous ERP project engagement, many interoperability challenges are real and burdening companies. For example, the coding for product modules and parts, which is a must for managing bills of materials in a collaborative environment, can be tricky; and a common protocol should be defined. The problem of identifying and hence further tracking or searching the required unique parts or module is widely reported. An alternative bill of material (BOM) management scheme that can be generically embedding or mapping with many different suppliers' schemes is a costly bottleneck problem. Here is a real example challenge that highlights the importance of system compatibility. The ordering company uses a set of coding system for product part identification while the suppliers use different systems; part identification becomes a complex problem. This is exactly a recent difficulty that the collaborating company has just encountered. A sustainable coding system for the collaborative practice has to be adopted; therefore the design and schematically specifications of such a coding scheme need research effort in the field of manufacturing collaboration lifecycle analysis.

Fourth, develop generic and feature-based cost engineering functionality [9, 10]. If the access to precise product and process information for fulfilling potential orders is available, companies can utilize necessary optimization strategies to work out the accurate and timely cost and delivery schedule estimation. The proposed innovative mechanisms will allow company to be more responsive to business opportunities and order delivery.

The proposed research project deliverables are: (1) A set of acceptable and generic manufacturing outsource and in-house process models and documented implementation procedures in a form of standardized operation manuals and the corresponding computerized management interfaces integrated with the managing ERP system; (2) A prototyping software toolkit that can generate complete neutral data file schemas required for interfacing different ERP packages for migration/integration such that the new ERP system can access and use ERP data contents 
effectively from different sources; (3) A tested effective working procedure and a working computer program that can be integrated with the new ERP software so that different scenarios of cost engineering in the context of supply chains can be quantitatively simulated and compared.

These deliverables are the coherent elements of the ERP adoption or upgrading research; the resulted findings can be applied to many companies in the manufacturing sector. Companies can apply the methodology for ERP implementation for the first time in a collaborative manufacturing environment, or for ERP system integration or upgrading. The neutral process data model has a long term impact to the industry because companies need such a standard sooner or later if they don't want to be bound by one single package forever. The key research innovation for the proposed framework_should be on the generic data extraction methods and the necessary constraint solving mechanisms.

\section{Expected Research Activities}

There have been many commercial ERP tools [11] implemented in companies, but advanced research on flexibility and adaptation effectiveness is still insufficient due to the business model transitions and customer demand changes [12]. Representatively, in order to achieve the transition from typical in-house manufacturing business model into an open and collaboration-oriented collaborative manufacturing model, the author suggests starting with the development of consistent ERP data models. The research activities would include manufacturing process modeling and redevelopment of the ERP interfaces for the new scenarios with the distributed manufacturing management requirement; automated product model searching and traversing algorithms, generic computational solution for the resources planning and job scheduling in a dynamic supply chain system; neutral data schema definition for the automated ERP data migration or integration, and finally, the development of a cost engineering method under the new framework of ERP implementation. Then, the research effort should develop the generic and acceptable manufacturing outsource and in-house process models and procedures in the form of standardized operation data forms and computerized management interfaces integrated with the new ERP system. Next, working software modules to generate and modify master production schedule with the consideration of available capabilities and resources are to be designed and prototyped. Implementation is not a difficult task which can be contracted to consulting service companies. Tests should be done to ensure those updated or migrated data files generated by different ERP tools can be accessed and used effectively by the master ERP system. A set of effective working procedures and proven mechanisms should be developed and documented. For example, different scenarios of cost engineering in the context of supply chains can be quantitatively simulated and compared.

\section{Summary}

This paper proposes a new ERP implementation approach, where complex product assembly structures and configurations as well as the dynamic manufacturing processes are integrated via a unified feature modeling method; data models, procedures and communication forms are to be formalized.

\section{Acknowledgement}

The author would like to acknowledge the funding support from a Canada NSERC discovery grant (No. 355454-09) and MITACS industrial internship cluster program. He acknowledges the collaboration support from McCoy Drilling and Completions - Farr in Edmonton, Alberta, Canada. The author also appreciates very much the assistance from his MSc students, Ms. Narges Sajadfar and Mr. Jingxing Wei, for their related research effort. 


\section{References}

[1] B. Grabot, et al., ERP Systems and Organizational Change: A Socio-technical Insight, Springer, London, 2008.

[2] S.V. Grabski, et al., Risks and Controls in the Implementation of ERP Systems, Int. J. of Digital Acc. Res., 1 (2001) 47-68.

[3] D. Aloini, R. Dulmin, V. Mininno, Risk Management in ERP Project Introduction: Review of Literature, Infor. and Manag., 44 (2007) 547-567.

[4] Z.M. Bi, S.Y.T. Lang, W. Shen, and L. Wang, Reconfigurable manufacturing systems: the state of the art, Int. J. of Prod. Res. 46 (2007) 967-992.

[5] C.H. Kuei, C.N., Madu, C.H. Lin, Developing global supply chain quality management systems, Int. J. of Prod. Res, 49 (2011) 4457-4481.

[6] Y.-S. Ma (editing author), Semantic Modeling and Interoperability in Product and Process Engineering - A technology of engineering informatics, Springer, London, 2013, new book.

[7] J. Wei and Y.-S. Ma, Design of an Order Acceptance and Scheduling Module in a Unified Framework with Product and Process Features, in Proc. of IEEE CASE12, Seoul, 968-973.

[8] J. Wei and Y.-S. Ma, Design of a Feature-based Order Acceptance and Scheduling Module in an ERP System, accepted by Computers in Industry, Elsevier, to appear.

[9] Y.-S. Ma, N. Sajadfar and L. Campos Triana, A feature-based semantic model for automatic product cost estimation, accepted by the 3th World Conference on Science and Engineering (WCSE2013), Singapore, August 24 - 25, 2013, and to be available in Int. J. of Eng. and Tech.

[10] N. Sajadfar, L. Campos Triana and Y.-S. Ma, Interdisciplinary semantic interactions within a unified feature model for product cost estimation", accepted in the Proc. of the Int. Conf. on Mech. Eng. and Mechatronics, Toronto, August 8-10, 2013.

[11] D.L. Olson, B.K. Chae, C. Sheu, Relative impact of different ERP forms on manufacturing organizations: an exploratory analysis of a global manufacturing survey, Int. J. of Prod. Res., 51 (2013) 1520-1534.

[12] J.H. Worley, K.A. Chatha, R.H. Weston, O. Aguirre, B. Grabot, Implementation and optimisation of ERP systems: A better integration of processes, roles, knowledge and user competencies, Comp. in Ind. 56 (2005) 620-638. 